\title{
AVALIAÇÃO DA ESTABILIDADE OXIDATIVA DE BIODIESEIS PRODUZIDOS A PARTIR DE ÓLEOS VEGETAIS
}

\author{
L. G. LENHARD ${ }^{1}$, M. C. MILINSK ${ }^{1}$, D. C. LENHARD ${ }^{2}$ e G. GOMES ${ }^{1}$ \\ ${ }^{1}$ Universidade Federal do Paraná, Curso de Tecnologia em Biocombustíveis \\ ${ }^{2}$ Universidade Tecnológica Federal do Paraná, Núcleo de Ciência de Alimentos \\ E-mail para contato: daiane_lenhard@yahoo.com.br
}

\begin{abstract}
RESUMO - O trabalho teve como objetivo avaliar a estabilidade oxidativa de óleos vegetais e dos biodieseis produzidos com estes óleos. Para produção dos biodieseis foi utilizado óleo de canola, óleo de soja e óleo de linhaça in natura, e os mesmos óleos aquecidos a $120^{\circ} \mathrm{C}$ por $2 \mathrm{~h}$. Os óleos utilizados para produção dos biodieseis apresentaram ácidos graxos insaturados, dos quais quimicamente são mais propensos a agentes oxidantes, podendo causar mudanças físico-químicas na composição do óleo. O biodiesel por ser oriundo desses óleos, pode apresentar características indesejáveis que quando incorporadas ao biodiesel prejudicam a qualidade do mesmo. Os biodieseis foram produzidos utilizandose uma proporção de óleo:metanol de 1:6 e concentração do catalisador, $\mathrm{NaOH}$, de 0,5 mol. $\mathrm{L}^{-1}$. Tanto os biodieseis quanto os óleos, foram analisados quanto ao índice de acidez e índice de peróxido (a partir de método titulométrico) e extinção específica na região ultravioleta (a partir de método instrumental, utilizando espectrofotômetro). No caso dos biodieseis, as três análises foram realizadas em intervalos de dez dias, durante dois meses, a fim de se verificar a influência do tempo de estocagem na oxidação do biodiesel. Foram observadas diferenças nas análises físico-químicas das amostras, mostrando que as mesmas sofreram oxidação durante os intervalos de estocagem.
\end{abstract}

\section{INTRODUÇÃO}

Os lipídios, principais constituintes dos óleos e gorduras são substâncias lipofílicas bastante utilizadas pelos organismos vivos como forma de armazenamento de energia, fontes de ácidos graxos e como veículos no transporte das vitaminas lipossolúveis e são conhecidos desde os ancestrais pela fácil extração e possuir diversas propriedades (Masuchi et al., 2008). Os ácidos graxos são ácidos carboxílicos com cadeias hidrocarbônicas de comprimento entre 4 e 36 carbonos $\left(\mathrm{C}_{4}\right.$ e $\left.\mathrm{C}_{36}\right)$. Podem ainda apresentar cadeias saturadas, ou seja, não possuem duplas ligações e ramificações, como também cadeias com uma ou mais dupla ligações (Nelson et al., 2002).

A degradação de um óleo depende da presença de ácidos graxos insaturados em sua composição. Óleos vegetais que possuem uma grande quantidade de ácidos graxos insaturados são mais suscetíveis aos ataques de agentes oxidantes como os radicais livres, as enzimas e metais. E as principais alterações que ocorrem nos óleos são por processos químicos como a auto-oxidação, a polimerização térmica ou a oxidação térmica, que podem ser acelerados pelo 
calor, luz (foto-oxidação), ionização, traços de metais ou catalisadores (Fonseca e Yoshida, 2009).

A reação de oxidação pode iniciar-se nos pontos alílicos as duplas ligações. Assim ácidos graxos como oléico (C18:1n9), linoléico (C18:2n6) e linolênico (C18:3n3) são mais suscetíveis a oxidação e fazendo parte do biodiesel, torna-o relativamente instável, formando produtos residuais como, ácidos e aldeídos causados pela degradação podendo interferir na qualidade e no seu funcionamento (Sarin et al., 2010).

Diferentemente dos combustíveis fósseis, que são relativamente inertes e mantêm as suas características essenciais pouco alteradas, o biodiesel degrada-se com o tempo devido ao seu contato com contaminantes. Dessa forma, manter a qualidade do biodiesel e as misturas com o diesel pode ser um desafio que está ligado diretamente aos produtores, vendedores e usuários (Galvão, 2007).

Existem técnicas clássicas de análises da qualidade de óleos e gorduras, como índices de peróxidos e acidez, que podem ser aplicadas ao biodiesel como forma de avaliar estabilidade a oxidação deste produto. No entanto, esses métodos demandam certo tempo e não fornecem com clareza as alterações moleculares produzidas no processo. Porém, mesmo que os resultados obtidos apresentem baixa confiabilidade e seletividade, estes parâmetros podem auxiliar no processo reacional e na avaliação oxidativa durante o tempo de estocagem de maneira simples e econômica (Melo, 2010).

O índice de acidez pode ser utilizado para medir a quantidade formada de ácidos graxos livres, decorrentes de uma hidrólise parcial dos triacilgliceróis, sendo definida em quantidade de gramas de ácido livre para cada $100 \mathrm{~g}$ de amostra analisada. Com relação ao teor de peróxidos, este pode ser utilizado como um indicador de que sua amostra está sofrendo oxidação, no entanto, quando a amostra apresenta baixo índice de peróxidos, dificulta-se a visualização final do ponto de titulação, limitando sua determinação sua nos estágios iniciais de oxidação. No entanto, níveis baixos de peróxidos, não implicam que a amostra possua uma boa estabilidade oxidativa, podendo ser, uma alteração pronunciada (Melo, 2010).

As análises de índice de peróxido e dienos conjugados utilizadas para acompanhar a evolução da oxidação nos métodos acelerados em alta temperatura apresentam valor questionável, pois os peróxidos se decompõem rapidamente em temperatura elevada. A formação de hidroperóxidos e dienos conjugados está relacionada à baixa na concentração de $\alpha$-tocoferóis. Logo, a formação de hidroperóxidos e dienos conjugados são alvos fáceis de ataques por radicais livres, formados sob altas temperaturas, deterioram o óleo, tornando-o impróprio para o consumo (AntoniassI, 2001; Reda, 2004). A manutenção da qualidade do biodiesel, necessária para difundir seu uso como combustível alternativo depende do desenvolvimento de tecnologias que aumentem sua resistência à oxidação durante longos tempos de estocagem (Ferrari et al., 2005). 
Assim, o presente estudo teve por objetivo avaliar a estabilidade oxidativa do biodiesel obtido a partir de diferentes óleos vegetais (canola, soja e linhaça) in natura e aquecidos, através das análises físico-químicas, após determinados tempos de estocagem.

\section{MATERIAIS E MÉTODOS}

Para este experimento foram utilizados três óleos vegetais: soja (OS), canola (OC) e linhaça (OL), adquiridos no comércio local. Os óleos foram divididos em frascos âmbar com capacidade de $500 \mathrm{~mL}$ cada. Cada óleo foi distribuído em dois frascos com volumes iguais, sendo três armazenados in natura (OS_N, OC_N e OL_N) e os demais aquecidos a $120^{\circ} \mathrm{C}$ por 2 horas (OS_A, OC_A e OL_A). Esses óleos foram selecionados devido à diferença na concentração dos principais ácidos graxos presentes: oléico (C18:1n-9), linoléico (C18:2n-6) e $\alpha$ - linolênico (C18:3n-3).

A produção do biodiesel foi realizada utilizando as seis amostras de óleos supracitadas, cujas denominações estão descritas no item 5.

Todas as análises realizadas nos biodieseis produzidos foram realizadas em intervalos de tempo de 15 dias, totalizando um período de análise de dois meses.

\subsection{Processo de Aquecimento dos Óleos}

O óleo foi transferido para um béquer com capacidade para $600 \mathrm{~mL}$ e permaneceu sob aquecimento por 2 horas com a temperatura em torno de $120^{\circ} \mathrm{C}$, em uma chapa de aquecimento. Após esta etapa, o óleo foi mantido em repouso até atingir a temperatura ambiente e em seguida armazenado em frasco âmbar. Este procedimento foi realizado com os óleos de soja, canola e linhaça selecionada obtendo-se as amostras OS_A, OC_A e OL_A, respectivamente.

\subsection{Reação de Transesterificação}

O procedimento para obtenção do biodiesel foi realizado mantendo a proporção de 1:6 (óleo:metanol) e concentração do catalisador $(\mathrm{NaOH})$ de $0,5 \mathrm{~mol} . \mathrm{L}^{-1}$ em metanol. Foram pesados aproximadamente $200 \mathrm{~g}$ de óleo em um béquer e adicionados a solução de $\mathrm{NaOH} 0,5 \mathrm{~mol} . \mathrm{L}^{-1} \mathrm{em}$ metanol. A mistura foi mantida sob agitação por 30 minutos a temperatura ambiente. Após, foi transferido para um funil de separação e deixado em repouso para separação das fases. A fase superior foi coletada e em seguida, destilado o metanol residual.

\section{3.Índice de Acidez}

O índice de acidez (IA) foi determinado de acordo com o método proposto pela AOCS Ca 5a-40 (1998) e calculado conforme a Equação 1. Foram pesados em um erlenmeyer aproximadamente 2,0 g da matéria prima (OS_N, OS_A, OC_N, OC_A, OL_N, OL_A). Em seguida, adicionados 25,0 mL de uma solução de éter - álcool etílico (2:1) e agitado. Foram 
adicionadas 2 gotas do indicador fenolftaleína. Titulou-se com uma solução de hidróxido de sódio 0,01 mol. $\mathrm{L}^{-1}$ até o aparecimento da coloração rosa.

$$
I A=\frac{5,61 \cdot V \cdot C \cdot f c}{m}
$$

\section{4. Índice de Peróxido}

O Índide de Peróxido (IP) foi determinado conforme o método descrito por Adolfo Lutz (2008) e calculado conforme a Equação 2. Foram pesados aproximadamente 5,0 g da amostra em um erlenmeyer de $250 \mathrm{~mL}$. Nestes foram adicionados $30 \mathrm{~mL}$ da solução de ácido acéticoclorofórmio (3:2 v/v) e agitados até a dissolução da amostra. Adicionou-se 0,50 mL de uma solução saturada de iodeto de potássio e a mistura reacional foi mantida em repouso e ao abrigo da luz por um minuto. Em seguida, adicionou-se $30 \mathrm{~mL}$ de água e titulou-se com solução de tiossulfato de sódio $0,01 \mathrm{~N}$ até que o desaparecimento da coloração amarela. Após, adicionou-se 0,5 mL de solução de amido $1 \%$ procedendo-se a titulação até o completo desaparecimento da coloração azul. Para efetuar os cálculos realizou-se o procedimento em uma prova em branco.

$$
I P=\frac{1000 \cdot(A-B) \cdot N \cdot f c}{m}
$$

\section{RESULTADOS E DISCUSSÃO}

Os dados apresentados nas tabelas que se seguem, estão expressos como média \pm desvio padrão das medidas em triplicata.

Os resultados referentes ao índice de acidez dos óleos vegetais analisados estão apresentados na Tabela 1 .

Tabela 1 - Índice de acidez dos óleos vegetais

\begin{tabular}{ll}
\hline Amostras & IA $(\mathrm{mg} \mathrm{KOH} / \mathrm{g})$ \\
\hline OS_N & $0,028 \pm 0,0012$ \\
OS_A & $0,027 \pm 0,0000$ \\
OC_N & $0,030 \pm 0,0004$ \\
OC_A & $0,029 \pm 0,0012$ \\
OL_N & $0,152 \pm 0,0015$ \\
OL_A & $0,153 \pm 0,0058$ \\
\hline
\end{tabular}

Os valores obtidos para o IA dos óleos apresentou-se dentro do permitido segundo a ANVISA, sendo $0,6 \mathrm{mg} \mathrm{KOH} / \mathrm{g}$ para óleos refinado. Os maiores valores apresentados foram para o óleo de linhaça. Este fato pode estar relacionado com o tipo de extração a qual o mesmo foi obtido, a prensagem a frio e por não ser refinado. Estes processos podem levar a obtenção de um 
óleo com presença de impurezas as quais podem levar a rancidez hidrolítica. No entanto, de acordo com a ANVISA, o valor máximo de IA para óleos obtidos por prensagem a frio deve ser de 4,0 mg KOH/g, assim o mesmo encontra-se dentro do recomendado (ANVISA, 2005).

Na Tabela 2 estão apresentados os dados referentes às análises de IA e IP do biodiesel do óleo de canola in natura e aquecido.

Tabela 2 - Parâmetros analisados no biodiesel produzido a partir do óleo de canola em diferentes intervalos de tempo de estocagem

\begin{tabular}{ccccr}
\hline \multicolumn{2}{c}{ BC_N } & \multicolumn{2}{c}{ BC_A } \\
\hline $\begin{array}{c}\text { Tempo de } \\
\text { estocagem } \\
\text { (dias) }\end{array}$ & $\begin{array}{c}\text { IA } \\
(\mathrm{mg} \mathrm{KOH} / \mathrm{g})\end{array}$ & $\begin{array}{c}\text { IP } \\
\left(\mathrm{meq} \mathrm{O}_{2} / \mathrm{Kg}\right)\end{array}$ & $\begin{array}{c}\text { IA } \\
(\mathrm{mg} \mathrm{KOH} / \mathrm{g})\end{array}$ & $\begin{array}{c}\text { IP } \\
\left(\mathrm{meq} \mathrm{O}_{2} / \mathrm{Kg}\right)\end{array}$ \\
\hline 0 & $0,023 \pm 0,0013$ & ------- & $0,023 \pm 0,0013$ & ------ \\
15 & $0,036 \pm 0,0013$ & $3,995 \pm 0,1406$ & $0,040 \pm 0,0022$ & $3,995 \pm 0,1402$ \\
30 & $0,035 \pm 0,0000$ & $5,794 \pm 0,1409$ & $0,042 \pm 0,0013$ & $7,093 \pm 0,1393$ \\
45 & $0,053 \pm 0,0033$ & $8,688 \pm 0,4214$ & $0,061 \pm 0,0012$ & $8,491 \pm 0,5665$ \\
60 & ------- & $11,49 \pm 0,2854$ & ------- & $12,09 \pm 0,1348$ \\
\hline
\end{tabular}

Verifica-se que houve um aumento gradativo dos índices de acidez e peróxidos nas duas amostras, sendo um indicativo que as amostras podem ter sofrido algum processo de oxidação.

Os resultados referentes às análises realizadas no biodiesel proveniente do óleo de soja in natura e aquecidosestão apresentados na Tabela 3.

Tabela 3 - Parâmetros analisados no biodiesel produzido a partir do óleo de soja em diferentes intervalos de tempo de estocagem

\begin{tabular}{ccccc}
\hline \multicolumn{2}{c}{ BS_N } & \multicolumn{2}{c}{ BS_A } \\
\hline $\begin{array}{c}\text { Tempo de } \\
\text { estocagem } \\
\text { (dias) }\end{array}$ & $\begin{array}{c}\text { IA } \\
(\mathrm{mg} \mathrm{KOH} / \mathrm{g})\end{array}$ & $\begin{array}{c}\mathrm{IP} \\
(\mathrm{meq} \mathrm{O} / \mathrm{Kg})\end{array}$ & $\begin{array}{c}\text { IA } \\
(\mathrm{mg} \mathrm{KOH} / \mathrm{g})\end{array}$ & $\begin{array}{c}\text { IP } \\
\left(\mathrm{meq} \mathrm{O}_{2} / \mathrm{Kg}\right)\end{array}$ \\
\hline 0 & $0,027 \pm 0,0005$ & $12,48 \pm 0,2804$ & $0,030 \pm 0,0012$ & ------- \\
15 & $0,039 \pm 0,0012$ & $17,28 \pm 0,1374$ & $0,038 \pm 0,0000$ & $7,992 \pm 0,3694$ \\
30 & $0,049 \pm 0,0013$ & $27,68 \pm 0,5184$ & $0,044 \pm 0,0013$ & $16,78 \pm 0,0042$ \\
45 & $0,060 \pm 0,0034$ & $32,09 \pm 0,4264$ & $0,053 \pm 0,0045$ & $24,17 \pm 0,5630$ \\
60 & --------- & $38,86 \pm 0,1501$ & ------ & $30,57 \pm 0,2405$ \\
\hline
\end{tabular}

Com relação aos resultados obtidos para o biodiesel produzido a partir do óleo de soja in natura, o índice de peróxido apresentou um aumento acentuado nos intervalos de análises. Estes dados mostram que o tempo de estocagem pode levar a oxidação do biodiesel. Outro fator a ser considerado nestes resultados deve ser o processo de destilação pelo qual esta amostra de 
biodiesel foi submetida. A amostra foi submetida várias vezes ao processo de aquecimento para remoção do excesso metanol, podendo ter ocasionado alterações químicas, levando a oxidação.

No que diz respeito ás análises para o biodiesel produzido a partir do óleo aquecido,observou-se um aumento gradativo tanto do índice de acidez, quanto e peróxido, verificando que o mesmo pode estar sofrendo processo de oxidação.

As análises realizadas no biodiesel proveniente do óleo de linhaça in natura e aquecido estão apresentadas na Tabela 4.

Tabela 4 - Parâmetros analisados no biodiesel produzido a partir do óleo de em diferentes intervalos de tempo de estocagem

\begin{tabular}{ccccc}
\hline & \multicolumn{2}{c}{ BL_N } & BL_A \\
\hline $\begin{array}{c}\text { Tempo de } \\
\text { estocagem } \\
\text { (dias) }\end{array}$ & $\begin{array}{c}\text { IA } \\
(\mathrm{mg} \mathrm{KOH} / \mathrm{g})\end{array}$ & $\begin{array}{c}\mathrm{IP} \\
\left(\mathrm{meq} \mathrm{O}_{2} / \mathrm{Kg}\right)\end{array}$ & $\begin{array}{c}\text { IA } \\
(\mathrm{mg} \mathrm{KOH} / \mathrm{g})\end{array}$ & $\begin{array}{c}\text { IP } \\
(\mathrm{meq} \mathrm{O} / \mathrm{Kg})\end{array}$ \\
\hline 0 & $0,037 \pm 0,0013$ & -------- & $0,039 \pm 0,0013$ & ------- \\
15 & $0,050 \pm 0,0026$ & ------- & $0,057 \pm 0,0025$ & $3,895 \pm 0,0018$ \\
30 & $0,058 \pm 0,0013$ & $5,397 \pm 0,4256$ & $0,060 \pm 0,0013$ & $7,494 \pm 0,2455$ \\
45 & $0,082 \pm 0,0033$ & $8,192 \pm 0,3733$ & $0,082 \pm 0,0034$ & $8,791 \pm 0,3716$ \\
60 & -------- & $12,09 \pm 2,079$ & --------- & $2,49 \pm 0,6991$ \\
\hline
\end{tabular}

O óleo de linhaça por ser composto por em maior percentual pelo ácido linolênico (18:3n3) tende a ser muito suscetível a oxidação. No entanto, apesar de ter apresentado maior acidez quando comparado aos demais, apresentou o menor nível de oxidação. Este fato pode estar relacionado à presença de antioxidantes naturais que ajudam a preservar a qualidade deste produto. Visto que a presença maior de insaturações não foi o fator crucial para levar a oxidação.

Os valores obtidos do BL_A foram muito próximos do BL_N, podendo ser considerado que o aquecimento utilizado não foi suficiente para modificar a estrutura química. Apresentando uma boa estabilidade em comparação as outras amostras.

O biodiesel obtido a partir de óleo de soja neutralizado pode apresentar maior estabilidade, devido ao fato de que possui em sua composição antioxidantes naturais, o que aumenta a estabilidade do óleo e também mostra efeitos benéficos sobre a retardamento da degradação oxidativa do biodiesel produzido a partir deste. No entanto, observou-se, que o biodiesel de óleo de soja in natura foi que apresentou maior índice de peróxido. Fato que pode estar relacionado com a etapa de destilação utilizada no processo de transesterificação. (Ferrari et al, 2005)

A elevação do índice de peróxido demonstra o aumento da oxidação térmica e lipídica, formando hidroperóxidos que podem comprometer o aroma, cor e sabor dos óleos, refletindo na qualidade do biodiesel, uma vez que esses óleos são incorporados para produção do biodiesel (Ramesh, 1995). 
Um elevado índice de acidez pode indicar que o biodiesel está sofrendo quebras em sua estrutura, liberando ácidos graxos livres, podendo ser provenientes de uma reação incompleta. No entanto, mesmo apresentando o maior índice de acidez, o óleo de linhaça ainda se encontra dentro dos parâmetros exigidos pela norma da ANP de 2012 (Alves, 2009).

\section{CONCLUSÃO}

Avaliando os dados referentes aos índices de acidez, peróxidos e extinção específica, verificou-se que o perfil em ácidos graxos pode ter influenciado nos resultados, no entanto, o óleo de linhaça que aparentemente poderia levar a um biodiesel mais propenso a oxidação foi o que se destacou em termos de qualidade. Este fato pode estar relacionado com o modo de obtenção do óleo (prensagem a frio) que não altera a quantidade de antioxidantes naturais presentes no óleo de maneira significativa. Assim, os parâmetros analisados mesmo sendo usados para medir a qualidade do óleo vegetal podem ser utilizados para avaliar a qualidade do biodiesel para prever o estado de oxidação do mesmo de maneira rápida e econômica.

\section{NOMENCLATURA}

A: Volume (mL) da solução de tiossulfato de sódio gasto na titulação

B: Volume $(\mathrm{mL})$ da solução de tiossulfato de sódio gasto na titulação do branco

BC_A: Biodiesel de óleo de canola após ser aquecido

BC_N: Biodiesel de óleo de canola in natura

BL_A: Biodiesel de óleo de linhaça após ser aquecido

BL_N: Biodiesel de óleo de linhaça in natura

BS_A: Biodiesel de óleo de soja após ser aquecido

BS_N: Biodiesel de óleo de soja in natura

C: Concentração de KOH (mol/L)IA: Índice de Acidez (mg de KOH/g)

fc: Fator de correção

IP: Índice de Peróxido (meq $\mathrm{O}_{2} / \mathrm{kg}$ )

m: Massa da amostra (g)

$\mathrm{N}$ :Concentração normal da solução de tiossulfato de sódio (eq/L)

OC_A:Óleo de canola após ser aquecido

OC_N: Óleo de canola in natura

OL_A: Óleo de linhaça após ser aquecido

OL_N: Óleo de linhaça in natura

OS_A: Óleo de soja após ser aquecido

OS_N: Óleo de soja in natura

$\mathrm{V}$ : Volume de $\mathrm{KOH}(\mathrm{mL})$

\section{REFERÊNCIAS}

ALVES, R. F.; GUIMARÃES, S. M.; ABREU T. C. e SILVA, R. D. Índices de Acidez Livre e de Peróxido. Relatório para a Disciplina de Bioquímica, Curso Técnico de Química 
Industrial, Centro de Educação Profissional Hélio Augusto de Sousa, São José dos Campos, SP, 2009.

ANTONIASSI, R. Métodos de avaliação da estabilidade oxidativa de óleos e gorduras. B. CEPPA, Curitiba, v. 19, n. 2, jul./dez. 2001.

ANVISA. Resolução $n^{\circ} 270$, de 22 de setembro de 2005. Regulamento técnico para fixação de identidade e qualidade de óleos vegetais, gorduras vegetais e creme vegetal, 2002.

FERRARI, R. A.; OLIVEIRA, V. S. e SCABIO, A. Oxidative stability of biodiesel from soybean oil fatty acid ethyl ester. Sci. Agric., v.62, Piracicaba, Brasil, 2005.

FONSECA, M. M. e YOSHIDA, M. I. Análise térmica do óleo de linhaça natural e oxidado. Vértices, Campos dos Goytacaz/RJ, v. 11, n. 1/3, p. 61-75, jan./dez. 2009

GALVÃO, L. P. F. C. Avaliação termoanalítica da eficiência de antioxidantes na estabilidade oxidativa do biodiesel de mamona. 161 f. Dissertação de mestrado em Química - Centro de Ciências exatas e da Terra, Universidade Federal do Rio Grande do Norte, Natal, 2007.

IAL - INSTITUTO ADOLFO LUTZ. Normas analíticas do Instituto Adolfo Lutz. Métodos Químicos e Físicos para Análise de Alimentos, v.1,3. ed. São Paulo: IMESP, 1985.

MASHUCI, M. L.; CELEGHINI, R. M. S.; GONÇALVES, L. A. G. e GRIMALDI, R. Quantificação de TBHQ (tercbutilhidroquinona) e avaliação da estabilidade oxidativa em óleos de girassol comerciais. Q. Nova, 31, 1053-1057, 2008.

MELO, M. A. M. F. Avaliação das Propriedades de Óleos Vegetais visando a Produção de Biodiesel. 118f. Dissertação (Mestrado em Química) - Centro de Ciências exatas e da natureza, Universidade Federal do Paraíba, João Pessoa, 2010.

NELSON, D. L; COX, M. e LEHNINGER, M. Princípios da Bioquímica. Terceira edição, agosto de 2002 .

RAMESH, M., Microwave treatment of groundnut (Arachis hipogaca): Extractability and quality of oil and its relation to lípase and lipoxygenase activity, Lebensmittel - Wissensechaft und - Technologie, v. 28, p. 96-99, 1995.

REDA, S. Y. Estudo comparativo de óleos vegetais submetidos a estresse térmico. 153f. Dissertação de mestrado em Engenharia de Alimentos- Setor de Ciências Agrárias Universidade Estadual de Ponta Grossa, 2004.

SARIN, A.; SINGH, N. P.; SARIN, R. e MALOTRA, R. K. Natural and synthetic antioxidants: Influence on the oxidative stability of biodiesel synthesized from non-edible oil. Energy, 2010. 